position that the pulse is a measurer neither of the speed nor quantity of blood circulated, nor of the power of the heart, is most evident when, assuming that the ventricle discharges the whole of its blood at each systole, it is considered that a pulse at 120 would, over a pulse at 60 , exhaust the labour of the heart by the square of the result. That the onward wave of the circulation does not constitute the pulse is evident, from the fact that, if a ligature be applied to an artery, the vibration of the pulse is felt both at the proximate and the distant ends of the vessel, where so tied, though the blood at these points forms no longer a portion of the moving fluid. The circulation is arrested, but the pulse continues.

The pulse is also felt more extensively in some cases in which the circulation is materially diminished, as where the blood becomes inspissated; or it may be even rendered more obvious and largely diffused, though the onward wave must be, comparatively speaking, disguised, or even lost entirely, as in an aneurismal dilatation. Here a fibrinous mass, which may scarcely admit a flow of blood through it, conımunicates on every side a hard and exaggerated pulse. The same may be observed when an artery is ossificd, or overlaid by a dense material, as also in those circumstances where elasticity compensates for, and obliterates, the shock of the onward wave; as occurs in the trunk artery and its larger branches, which offer but little external evidence, by expansion, of the onward wave, but freely communicate the vibrations of the pulse. The vibrations of the pulse are otherwise seen to be independent of the forward wave, for velocity in this may differ in different arteries, while the pulse retains its synchronous action. Again, where inflammation has induced a state of local congestion, the pulse may be, in the arteries concerned, more markedly felt. The above instances show conclusively, that the peculiar circumstance of pulsation, in whatever they may consist, is, in effect, separate from the blood-wave; and therefore it may be inferred it does not owe its immediate origin to that mechanical power or force which originates the circulation of the blood.

The blow which the heart makes against the ribs-the ictus ventriculi -and which does not occupy the period of the ventricular contraction, but only its concluding act, is neither a pulse nor the cause of it; not only are they not synchronous, but they bear little or no uniform relation in force to each other ; the former may be violent, the latter weak. Moreover, the various olsservations which have been made on the circum. stances of the circulating wave, show it not to be the result of a violent or spasmodic contraction of the originating power, such as might be inferred to be the case if it were the result of an action producing a violent rush of blood, and this so sudden as to be ejected at a single moment, and also to produce the vibration of the pulse. The experiments of Hales may be particularly referred to, in confirmation of the above; as also the absence of recoil in the distal arteries, and the absence of any bursting effect when, in an aneurism, the distal vessel is tied.

After fully considering the whole question, we can not but corclude that the circulating wave and the pulse are two separate and distinct operations, and therefore, as taking place in the same medium and transmitted by the same tubes, not due to the same cause. Doubtless, the contraction of the ventricle causes the projection of the column of blood, and this column traverses the arterial system by successive waves; while the pulse is a vibration taking place simultaneously through the whole particles of this, as it were, solid column of blood. The vibrations which constitute the pulse obey the same laws as sound transmitted in bodies, or as those vibrations which, when a blow is struck on one end of a log of wood, are felt distinctly by a hand applied to the other, although there be no visible locomotion. The pulse is, in fact, similar to that tangible shock which "is conveyed through a fluid without any apparent accumulation of it, or change of velocity. Thus, the working of a waterpump may be discovered at great distances through iron pipcs, or even through elastic pipes of leather, as those of a common fire-engine, from which the water is spouting nevertheless in a uniform stream," (Arnott).

What, then, is the cause of those vibrations which constitute the pulse, as separate from the circulation? In the course of the preceding argument it has been shewn that, in the arteries and in the moving column of fluid within them, the physical conditions exist for the ready transmission of vibrations occurring in this latter; and in a former paper it has been shewn, that the mechanism of the heart is well adapted for the production of these vibrations. (BRITISH MEDICAL JOURNAL, I th May, 1866, No. 288, p. 486.)

Having reference to that general law of hydraulics, which enunciates that the momentum of a liquid in motion is as great as that of a solid, and, hence, that anything which opposes or arrests this momentum, will receive as severe a blow as if from a solid, and cause within it the same disarrangement of particles, we see that, as the blood in circulation is a moving column, and its motion be opposed or arrested, there then exists the conditions for producing those vibrations, within the columns, similar to those which would take place in a solid. It has also been shewn (vide previous paper) that, in the sudden closure of the semilunar valves, the mechanism for thus opposing and arresting the circulation
exists.

It is probable, considering the elastic properties of the arteries, that, were it not for the closure of the semilunar valves during the diastole of the ventricle, whereby the momentum of the column of blood is arrested, the supply per saltum would have becn cqualised in these vessels, so that there would not only have bcen a pulseless wave, but one rendered tolerably even in its flow. As it is, the onward wave is, both in its origin and in its effect, independent of the vibrations which constitute the pulse, as also of the cause of these vibrations; while, on the other land, these vibrations are, in their origin, independent of the cause of the circulating wave. They are, in fact, dependent for their levclopment on that valvular mechanism, whereby the onward wave is suddenly arrested. It has been stated, that the passage of the blood through the arteries is characterised by three phenomena: 1 . the filling of the artery by the onward wave; 2 . the pulse; and 3 . the gradual cessation of vibration (vide ante); and looking to what has now been set forth, these phenomena may be thus explained. The moving wave, whereby the circulation of the wave takes place, is accounted for by the systole of the ventricle; then the sudden vibrations taking place simultaneously throughout the whole moving column of blood, and which constitute the shock or pulse, are accounted for by the momentum of this moving column being arrested by the sudden closure of the semilunar valves; and the succeeding pause is that period during which the momentum of the wave is temporarily arrested, and before it again has re-establishcd its even flow.

As regards the relations of the pulse to the sounds of the heart, in respect of time, it may be stated that the onward wave commences simultaneously with the first sound; the vibrations that constitute the pulse are synchronous with the second sound; and the pausc occupies the time between this and the first sound. The reading of the sphygmograph shows an ascending line; this commences synchronously with the first sound, while the highest point of this depictcd line is synchronous with the sccond sound, and the fall or wave-like decadence from this highest point, represents the pause or period between the second and first sounds. The explanation of all this is, that the ascending line of the sphygmograph depicts the expanding of the artery by the onward wave of the blood forced into it by the systole of the ventricle; its highest point, which is synchronous with the pulse-vibration, and with the second sound, represents the instant when the momentum of the onward current is arrested; while the fall in its line represents the period between the second and first sounds, and before the systolic action of the left ventricle has re-established the onward wave-flow.

Before concluding this paper, it may not be out of place to observe, that the theory of the pulse now set forth explains many peculiarities of the circulation observable in discase, and which, without it, are not, as it appears to me, easily to be cxplained; such as the violent beat of the heart with a feeble pulse; the absence of the true pulse-vibrations where valvular disease is confirmed; the undulations without pulse of the venous pulse; shock; etc. These will, however, be more particularly referred to when these diseased conditions are the especial subject of remark.

\section{REMARKS ON OBSTRUCTION OF THE BOWELS: WITH CASES.*}

By E. COPEMAN, M.D., Norwich.

CASE XXXvi.-Miss B., a delicate girl, about ig years of age, had been complaining now and then, for several weeks, of pain or uneasiness in the right iliac region, and was subject to constipation. About a week before 1 saw her she atc a good many nuts, and continued to eat some for two or three days afterwards, when she was suddenly seized with severe pain in the situation of the ileo-cæcal valve, and became sick and faint. After this she had no satisfactory relief from the bowels, although treated actively by her surgeon, and I was summoned on May I 4th, I863, in consultation. I found her at 5 P.M. with a depressed countenance, small rapid pulse, dry tongue, and considerable thirst. The abdomen was tumid and hard over the right iliac region, and tender; but there was no general peritonitis. She had vomited several times, and neither apcrients nor enemata had answered. I advised a discontinuance of the purgative plan; and we agreed to give her at once a large injection of soap and water and castor oil, to cover the abdomen with a bran poultice, and to give her a grain of the watery extract of aloes and a quarter of a grain of opium every three or four hours; soda water and milk to drink, and a little mutton broth occasionally, if not

- Continued from page 169 of vol. $\mathrm{i}$ for $\mathrm{I} 861$. 
sick. At 10 P.M., we found that the enema had passed away scarcely

tinged with fæces, the other symptoms remaining much the same.

May 15 th. - This morning we found the pulse 120 and a little improved in character, the tongue not so dry, and the skin moist. She did not vomit or feel sick during the night, and slept a good deal. The abdomen was not more distended, and was rather less painful; but there had been no relief from the bowels. We agreed to continue the treatment with the exception of reducing the quantity of opium to a sixth of a grain in each pill, and giving it not oftener than every four hours.

May 16th. - This morning we found her rather faint, and the pulse iveaker; but it was also slower (I08), which it would not have been had its weakness depended upon increased abdominal mischief. The cause of the depression seemed to be the occurrence of menstruation in the night, a fortnight before the proper time. In other respects she was easier; the abdomen was less tense and tender; but there had been no relief from the bowels. The pills were ordered to be continued every four hours. In the afternoon the bowels were relieved twice, and the dose of aloes was lessened for fear of purging.

May I 7th. - She had a good night, and slept so much that no pill had been given after midnight. There was no more relief from the bowels, but no sickness. Pulse I08; tongue furred. She felt altogether easier; and the abdomen, although firmer and more resisting than it ought to be in the situation of the ileo-crecal valve, was less distended and less tender. She was ordered a pill containing a grain of watery extract of aloes, to be taken alternately with those which she had before containing opium.

May I 8th. - She had another good night, and several dark green fluid motions. The abdomen was less tender; pulse 96 . The treatment was continued.

May 19th. - There were five or six moiions of the same colour and consistence in the last twenty-four hours, she having taken five of the pills in that time, three without and two with opium. The abdomen was still too resisting and tender to the touch, the left side being a little more painful than the right. Her general health was improved. She had no sickness; pulse 96. She took beef-tea and arrowroot with a little brandy.

May 23rd.-The same treatment had been continued to the present time, and she had been passing pulpy frces mixed with dark green bile from four to six times in twenty-four hours, without any irritation or purging, and with a gradual removal of swelling and tension in the original seat of pain. That part of the abdomen, however, was still rather tender, and there was evidently something more to pass. Her general health was daily improving; she took a little solid food yesterday. She took now three pills in twenty-four hours, one with opium and two without, and had a little mercurial liniment rubbed over the situation of the ileo-colic valve night and morning.

May 26th. - My colleague informed me to-day that on Sunday, the 24th, this patient passed nearly half a pint of pus by stool, but that she seemed to be much the same as before, having no increase of abdominal pain or fever, but on the contrary, less tenderness in the original seat of pain. From this period she slowly and gradually regained her former state of health, and had a satisfactory recovery.

CASE XXXVII.-On June 2nd, I863, I was called in consuitation to a delicate old lady, suffering from obstruction of the bowels, having had no relief for ten days. I found her with a very weak and frequent pulse, an anxious countenance, distended abdomen, and visible coils of bowe attempting to overcome the obstruction, which I imarined to be seated in the small intestines at or near the ileo-cæcal valve, where there was great tenderness on pressure. She had vomited frequently, and lately the ejecta were of a stercoraceous character. There was no sign of hernia in any of the usual situations. The surgeon in attendance had very carefully treated her in the usual manner with aperients of different kinds and enemata, but without any satisfactory results. recommended the injection of air into the bowel,

emergency grain doses of watery extract of aloes and opium every hour or two 1, the mouth. She had also turpentine stupes applied assiduously to the abdomen.

I saw her again on the 6 th, and found that since the injection of air she had passed a great deal of wind per anum, and all the general symptoms had subsided, although there had been no motion. She had not been sick since the day on which air had been injected; but as she complained a good deal of griping, we omitted the aloes, and gave her calomel and opium instead. Her pulse was down to 84, and her countenance was so improved as to denote, in conjunction with her generally amended condition, the removal of complete obstruction, although no motion had yet actually been passed. In a few days I was informed that free action of the bowels had taken place, and she gradually recovered.

CASE XXxviri.-On November 2nd, I863, I was requested to visit a lady of middle age, who had suffered from obstruction of the bowels for more than a week. There was evidently commencing peritonitis; the abdomen was greatly distended and very tender, the urine much loaded the pulse small and feeble, the tongue dry, countenance anxious, and she had frequent vomiting. I advised a grain each of calomel, watery extract of aloes, and opium to be taken every two hours, turpentine stupes to the abdomen, and a large enema in the morning if required.

Early on the following morning she had an enormous relief from the bowels; and from that time all danger was at an end, although she remained some time in a weak and feeble state, slowly recovering from the peritoneal mischief.

CASE XXXix. - On August Ioth, I865, I was summoned many miles into the country to visit a strong healthy-looking farmer, about 30 years of age, unmarried, living with his father, and leading a very active life. I found him suffering from serious obstruction of the bowels, stercoraceous vomiting, and a very tumid and painful abdomen. The symptoms were of more than a week's duration, and his medical man took it for a case of intussusception almost certain to lead to a fatal result; but there had been no discharge of bloody mucus or blood from the bowel, and I thought it a case of obstipation from some other cause. Purgatives had been freely used without effect, and no motion had passed for several days; we therefore agreed to discontinue them, and advised turpentine stupes, an enema of soap and water, and a pill containing a grain of watery extract of aloes and half a grain of opium every four hours.

On the I $3^{\text {th }}$, the surgeon wrote as follows. "Our patient is still alive, and I think, in some respects, a shade better. After we left him on Thursday evening, he had a great deal of pain and distension, for which a grain of opium was given at 6 P.M. At 10 he took another, with a grain of the extract of aloes, and so on during the night (one with opium alone, and the next with opium and aloes combined). On the following morning, I found him still in much pain, though not more than when you saw him. The rest of the injection had been passed, but without any feculent matter. He had taken a little milk two or three times in the night, and had no return of the sickness. I gave him another enema, and directed him to continue the pills. On Friday night I saw him again, and found him in more pain, with decided inflammatory symptoms arising in the right side. There was increased tenderness in the iliac region and along the cæcum. The pulse had risen to IOO, with a dry tongue. The enema returned alone; no sickness. I therefore ordered leeches over the painful part, and sent him some opium pills with half a grain of calomel instead of aloes. Yesterday morning he was decidedly relieved by the leeches; pulse down to 80; less pain on pressure, although still a good deal; no sickness. Moreover, he had passed a little wind per anum, which seemed to relieve him. I ordered six more leeches over the most painful part, to be followed, as before, by warm fomentations. Last night $I$ found him not so well again; the pulse had risen to 95 ; body very tympanitic; no more wind passed, but no return of sickness. This morning he was again easier, and had passed a little wind once or twice during the night, sufficiently odoriferous to have satisfied the old practitioner you referred to." (This was said in allusion to a remark I quoted, made by an old practitioner of my acquaintance some years ago, to the effect that no patient under such circumstances would die if he could pass wind per anum, although, quaintly, he expressed it in less refined terms.) "The pulse was down to 85; he had retained what milk he had taken, and the countenance does not look at present like that of a dying man. Still the body is very distended, and there is evidently as yet no free passage. I gave him another soap and water enema, and shall see him again this evening. It is a curious case, but not without hope; nor do I see that we can do better than persist in our present plan of treatment."

On the 17 th, I received the following letter. "You will be glad to hear that our patient is improving, although he has been very ill, and has had many relapses since I wrote on Sunday. On that evening and on Monday, the inflammatory action increased about the ileum and cxcum, with enormously distended abdomen, and no relief. Pulse 100 to I IO. The hopeful feature of the case, however, was that there was no return of sickness. He was leeched freely again on Sunday, Monday, and slightly on Tuesday, and always with marked benefit and relief of the pain. On Tuesday night he had a natural relief; on Wednesday another; and last night and this morning two or three relaxed, child. like motions; and this with his continued taking of five or six grains of opium in twenty-four hours, with two of calomel. His gums do not show the effect of the mercury; but as the inflammatory symptoms are subsiding, I have discontinued its use, and also diminished the opium. The tympanitis and remaining abdominal pain have also been relieved by turpentine stupes, which I ordered to be applied since the leeching has been discontinued. He has now commenced a more generous diet, eggs and milk, beef-tea, etc. At present there has been no appearance of blood in the stools. This has been an instructive case, and I am obliged to you for your advice and encouragement in its conduct." 
September 16th. - "I am glad to inform you that Mr. L. is quite recovered from his illness. He continued stendily to improve. I have not seen him for a fortnight, but he was thad "tucking in a good dinner of beef and bread, with port wine and water."

\section{REPORTS}

or

\section{MEDICAL AND SURGICAL PRACTICE IN THE HOSPITALS OF GREAT BRITAIN.}

COLDSTREAM GUARDS HOSPITAL.

FATAL CASE OF CHOREA IN THE ADULT MALE: AUTOPSY : WITH REMARKS.

(Under the care of Robert Farquharson, M.D., Assistant-Surgeon Coldstream Guards.)

THs following appears to be a well-marked example of that form of chorea which follows acute rheumatism and cardiac disease. Its in. terest is enhanced by the contrast it affords to the interesting case recently reported by Dr. Wilks (vide British Medical Journal, Feb. 29th), in which sudden fright was the exciting cause of the exaggerated movements.

J. A., aged 19, suffered in December 1862 from articular rheumatism, during which aortic regurgitant disease of the heart became developed. The bruit was at first unattended by symptoms; but pain, palpitation, and dyspncea gradually set in, and rendered him unfit to perform the duties of a soldier. On February I Ith, he had a slight relapse, which rapidly subsided under alkaline treatment; and the 16th found him tolerably free from pain. He was observed, however, to be restless and fidgetty, twitching his face and jerking his body; and he became rapidly worse until the 18th, when he presented the phenomena of chorea in their most aggravated form. It is difficult for language to give any idea of the excessive force and variety of the muscular contortions which had now taken complete possession of his frame. He perpetually tossed about, struggling against restraint, and every now and then seized with an almost tetanic spasm, bending the body into the form of a bow. Consciousness was not impaired; and he endeavoured to answer questions, but could not talk intelligibly. The movements continued with unabated vigour until next day, when they were observed to be en. tirely confined to the left side. This change, however, brought no relief to his sufferings, as he grew more violent than ever, and was with difficulty kept in bed by two powerful men. The urine was copious and limpid, specific gravity ror 3. Towards evening he became quieter, and apologised to those around him for the trouble he was giving; but this amendment was of brief duration. The choreic symptoms returned with great intensity; and death, preceded by rapid sinking, took place at 8 A.M.

The treatment included most of the principal sedative and narcotic remedies, which speedily lost their soothing influence on the nervous system. The hypodermic injection of morphia at first produced good results, and chloroform caused a few minutes' refreshing sleep; but it was found, on repetition, that the action of these drugs was unfortunately very transient. Lastly, cannabis indica was tried; but forty minims of the ethereal tincture caused so decided an aggravation of the symptoms of the disease, as to render any continuance of the experiment unjustifiable.

On post mortem examination, the pia mater covering the brain and anterior surface of the spinal cord was found to be abnormally vascular; and the right lateral sinus and internal jugular vein were gorged with dark blood. The anterior spinal artery and vein were of very large size; and some of the vessels accompanying the anterior roots of the spinal nerves appeared enlarged and tortuous. Naked-eye inspection detected nothing unnatural in the appearance or consistence of the nervous substance. The pericardium contained about four ounces of clear fluid. The heart was somewhat enlarged, and coated in places with recent lymph. Over a considerable portion of its outer wall and the corresponding pericardial surface, were scattered warty excrescences and roughened patches, giving the well-known resemblance to tripe. The valves were healthy, with the exception of the middle aortic, which was thickened by commencing vegetations. The other organs were free from disease. Some urine withdrawn from the bladder contained a large excess of urea.

REMARKS. - The main facts of this case seem so far to speak for themselves as to render comment unnecessary ; but I should like to draw attention to the very insidious manner in which the heart-disease stole on, without being attended by general symptoms sufficient to indicate its presence. That this occasionally happens in acute rheumatism, must be a matter of general observation; and I would throw out the hint, that the alkaline treatment, while undoubtedly lessening the risk of cardiac complications, may, by its lowering effect on the system, cause these to assume a masked form, if they do occur. I venture to make this suggestion, in consequence of having observed such a course of events in other cases of rheumatism which, like the preceding, had been treated by large does of alkalies.

In conclusion, I have to express my thanks to Surgeon-Major Cay for permission to publish these notes.

\section{ST. VINCENT'S HOSPITAL, DUBLIN.}

GANGRENE OF BOTH LITTLE TOES.

(Under the care of Dr. MA POTHER).

MARGARET BYRNe, aged 27, single, healthy, seven weeks since felt a severe pain in the right little toe; and, three days afterwards, matter formed under the nail and cuticle, which was evacuated by an incision. It was then found that a large slough had formed on the inner border of the toe. A fortnight afterwards, the left little toe became rapidly gangrenous to the level of the second phalanx, where a line of separation formed. She was then admitted to hospital. On July 9 th, the slough on the toe first attacked had come away; and Dr. Mapother proposed in a few days to remove the dead portion of the left toe by dividing the ligaments of the first phalangeal joint. No peculiarity in diet or health, and no local cause, could be charged with the production of this unusual symmetrical gangrene.

\section{ST. PANCRAS INFIRMARY.}

SENILE GANGRENE: DEATH: AUTOPSY.

(Reported by F. W. Gibson, M.D.Lond., Resident Medical Officer.) MARIA P., aged 75, was admitted on February 8th, 1868, on account of a superficial burn of the right foot and leg. On February 19th, gantrene of the second and third toes supervened, and spread about halfvay up the leg. No line of demarcation formed. She died on February 13rd. By post mortem examination, tubular calcification of the arteties of the limb, a fibrinous plug completely blocking up the lower part of the femoral and popliteal, fatty degeneration and general dilatation of the heart, atheroma of the aorta, great thickening of the capsule and trabeculæ of the spleen from fibrinous deposit, a granular condition of loth kidneys, and cirrhosis of the liver, were discovered.

\section{CHARING CROSS HOSPITAL.}

\section{SKIN-CLINIQUE : SYPHILITIC ACNE (ULCERATING) SIMULATING} LUPUS.

(Under the care of Dr. TIlbury Fox.)

THE following case is instructive in regard to ( 1 ) the differential diagnosis of lupus and syphilis, and (2) the treatment. The recognition of the disease as syphilitic demanded a plan of treatment wholly different from that which is called for in lupus, especially in reference to local applications.

The case was that of a single woman, C. W., aged 30, who was admitted Jan. 14th, 1868. As she entered the room, the paticnt seemed to present a large patch of ulcerating lupus, occupying the left side of the nose in its lower two-thirds. The patch had a dull red areola, but not of any very great extent; it did not sensibly exude; it was tolerably clean, and inclined to scab here and there. It was not painful, nor had it been so. The diseased surface, as a whole, was deprcssed below the level of the surrounding skin; and on closer inspection appeared to be uneven, being marked by depressions or pits, which were seen to be small ulcers, and in addition elevations, several of which were capped with little points. On questioning her, the following history was elicited. The woman suffered from primary syphilitic disease eight years since, which was neglected. This was followed by secondary mischief shortly afterwards. Five years ago "spots" appeared on the head, face, and legs; those on the latter were replaced by "ulcers." Scars now marked the site of those on the side of the head. The patient was at different times in St. George's, Charing Cross, and Westminster Hospitals for severe pains in different parts of her body, dependent apparently upon the syphilitic infection, for which she appears never to have been fairly treated. In one hospital she had her head shaved, was leeched on the scalp and temples, was blistered on the head and behind the ears, the blistered surface being kept open by the use of "green" 